KANSAS JOURNAL of MEDICINE

\title{
Evaluation of an Initial Specimen Diversion Device (ISDD) on Rates of Blood Culture Contamination in the Emergency Department
}

Blake A. Buzard, Pharm.D., Patrick Evans, Pharm.D., BCPS, Todd Schroeder, Pharm.D., BCPS (AQ-ID)

Ascension Via Christi St. Francis Hospital, Wichita, KS

Received June 22, 2020; Accepted for publication.Jan. 5, 2021; Published online March 19, 2021 https: / doi.org 10.17161 lijm.voll413804

\section{ABSTRACT}

Introduction. Blood cultures are the gold standard for identifying bloodstream infections. The Clinical and Laboratory Standards Institute recommends a blood culture contamination rate of less than $3 \%$. Contamination can lead to misdiagnosis, increased length of stay and hospital costs, unnecessary testing, and antibiotic use. These reasons led to the development of initial specimen diversion devices (ISDD). The purpose of this study was to evaluate the impact of an initial specimen diversion device on rates of blood culture contamination in the emergency department.

Methods. This was a retrospective, multi-site study including patients who had blood cultures drawn in an emergency department. February 2018 to April 2018, when an ISDD was not utilized, was compared with June 2019 to August 2019, when an ISDD was being used. The primary outcome was total blood culture contamination. Secondary outcomes were total hospital cost, hospital and intensive care unit length of stay, vancomycin duration of use, vancomycin serum concentrations obtained, and repeat blood cultures obtained.

Results. A statistically significant difference was found in blood culture contamination rates in the pre-ISDD group vs. the ISDD group (7.47\% vs. $2.59 \%, \mathrm{p}<0.001)$. None of the secondary endpoints showed a statistically significant difference.

Conclusions. Implementation of an ISDD reduced blood culture contamination. When implementing the ISDD to a healthcare system, compliance is important and will affect contamination rates dramatically. Kans J Med 2021;14:73-76

\section{INTRODUCTION}

Blood cultures are the gold standard for identifying bloodstream infections. However, blood cultures commonly become contaminated with environmental or skin-residing organisms. ${ }^{1,2}$ Contaminated cultures can lead to misdiagnosis, increased length of stay (LOS) and hospital costs, and unnecessary testing and antibiotic use. ${ }^{1-3}$ National contamination rate recommendations are set at less than $3 \%$ by the Clinical and Laboratory Standards Institute (CLSI). However, institutions across the U.S. have varying and often higher contamination rates ranging from 2 - 10\%. Ascension Via Christi Hospitals, Inc (AVC) ministries includes three separate hospitals: St. Francis, St. Joseph, and St. Teresa. St. Francis is considerably larger and is a Level 1 trauma center with access to multiple specialties. St. Joseph and St. Teresa are smaller tertiary hospitals with less resources and support including phlebotomy access. Across all AVC ministries, average contamination rates are about $6 \%$ in blood draws occurring in the emergency department (ED).

There are many organisms that can lead to contaminated blood cultures, but among the most common are coagulase-negative staphylococci, Corynebacterium species, Bacillus species other than Bacillus anthracis, Propionibacterium acnes, Micrococcus species, viridans group streptococci, and Clostridium perfringens., ${ }^{1,2,3}$ All of these pathogens can represent true bacteremia when found in a blood culture. Obtaining accurate blood cultures will prevent potential errors in diagnosing, unwarranted lab tests, antibiotic usage, and lower total costs hospital wide. Blood culture contaminants increase total hospital costs from $\$ 4,500$ - 13,000 per contaminant. ${ }^{2,4,6,7}$ For the current study, the median of the existing data of $\$ 8,750$ per contaminant was used. Of note, several of the existing financial outcome studies were older, with one of them being from over a decade ago. ${ }^{6}$ The median number utilized may not reflect the inflation or increased hospital costs that have occurred during that time span. It also did not reflect the change in price for the actual device.

There are many practices that decrease the number of blood culture contaminants, which include pulling from independent venipuncture sites, use of alcohol or chlorhexidine swabs prior to puncture, and use of highly-trained phlebotomists. ${ }^{3}$ Gander et al. ${ }^{6}$ evaluated the advantage of utilizing phlebotomists for all blood culture draws in the ED versus regular nursing draws. The study showed a decrease of contamination from $7.1 \%$ to $3.1 \%$ using phlebotomists. However, this is above the national benchmark of less than $3 \%$ contamination.

AVC ministries use a variety of practices when sepsis is suspected. AVC utilizes rapid diagnostic tests to identify gram positive organisms, especially Staphylococcus and Streptococcus species, within a matter of hours. If sepsis is suspected, patients go through standard practices regarding fluid requirements and antibiotics within one hour in accordance with guidelines. Alcohol swabs are used in the emergency departments for venipuncture sites, as opposed to chlorhexidine or povidone-iodine swabs. AVC contamination rates have varied when looking specifically at phlebotomist drawn labs, but the range varies from 1 - $4 \%$ month to month. Consistently, phlebotomy drawn blood cultures have contamination rates that are closer to or are under the national goal of less $3 \%$. Phlebotomy is not used within any of the AVC ministry emergency departments; thus, this study population did not capture any benefit from phlebotomy use.

There are other factors that affect contamination rates as well, including skill level of the staff and educational interventions. In ED environments, it can be challenging to monitor technique and re-educate when needed. ${ }^{1}$ To combat these challenges, two initial specimen diversion devices (ISDD) were produced. Steripath ${ }^{\mathrm{TM}}$ is a device that mechanically diverts and sequesters $1.5-2 \mathrm{~mL}$ of blood, which commonly is considered the volume that most likely contains skin-residing organisms. It allows for a separate, sterile blood flow pathway to be collected in a closed vein-to-vial path. This was the device piloted during the study. Kurin ${ }^{\mathrm{TM}}$ sequesters the first small amount of blood, but this volume is closer to $0.1-0.2 \mathrm{~mL}$ of blood. ${ }^{8}$ This device also does not use 
a separate, sterile pathway as opposed to the Steripath ${ }^{\mathrm{TM}}$ device. The use of ISDD's can lower contamination rates by $80 \%$ of the baseline contaminant rate and to a total contamination rate to less than $1 \%{ }^{1,4,9}$

AVC chose to implement a three-month pilot use of Steripath ${ }^{\mathrm{TM}}$ for blood cultures drawn in the ED. The three-month time period was compared to another three months from the year prior.

With a decrease of contaminated blood culture rates by $80 \%, \mathrm{AVC}$ would be below the national recommended rate of less than $3 \%$. For this study, an initial specimen diversion device was evaluated to determine its effectiveness in lowering the contamination rates of blood cultures drawn in the ED.

\section{METHODS}

This study was approved by the institutional review board as a retrospective chart review conducted at Ascension Via Christi Hospitals, Inc., that included patients in the emergency department who had a blood culture collected. The time frame in which an ISDD was used for collection was June 1, 2019 to August 31, 2019. This time frame was compared to February 1, 2018 to April 31, 2018 in which an ISDD was not used. Historically, there were ongoing issues with higher contamination rates despite implementing different practice changes. The two time frames were chosen specifically as it was confirmed the same practices were used to gather blood cultures and no other practice changes were ongoing during the separate time periods. There was little nursing turnover within the emergency department during this time period, with the majority of the same nurses included within both time periods. Patients were identified from the electronic health record (EHR) if a blood culture was taken in the ED during those two time periods. Patients who met inclusion criteria were divided into their respective groups and analyzed for the primary and secondary endpoints (Table 1). The primary endpoint was total blood culture contamination rate. The secondary endpoints analyzed were total hospital cost, hospital length of stay, intensive care unit (ICU) length of stay, vancomycin duration of use, vancomycin serum concentrations obtained, and repeated blood cultures obtained. The cost for the device used in the analysis was \$15 per device.

Table 1. Inclusion and exclusion criteria.

\begin{tabular}{|l|l|}
\hline Inclusion Criteria & Exclusion Criteria \\
\hline Age $\geq 18$ years of age & Age $<18$ years of age \\
\hline Blood culture obtained in the ED & \\
\hline
\end{tabular}

Outcomes. The primary endpoint was total blood culture contamination rate. The secondary endpoints analyzed were total hospital cost, hospital length of stay, ICU length of stay, vancomycin duration of use, vancomycin serum concentrations obtained, and repeated blood cultures obtained. The ISDD group had a primary endpoint analysis in both an intent-to-treat manner and per protocol manner. The per protocol examination excluded all contaminants from the ISDD group in which the ISDD was confirmed not to be used. For the secondary endpoints, hospital length of stay and ICU length of stay were not included if the stay was greater than seven days. The time frame was established to avoid including patients in the hospital staying for longer periods due to reasons other than a blood culture contaminant. Repeated blood cultures taken greater than seven days from the original blood cultures
KANSAS JOURNAL of MEDIC INE INITIAL SPECIMEN DIVERSION DEVICE

continued.

also were excluded from the study. The time frame was established to avoid capturing repeated blood cultures obtained for reasons other than repeating a blood culture after a contaminant.

Statistical Analysis. To detect an $80 \%$ reduction in the two groups with an $\alpha<0.05$ and a power of $0.80,235$ subjects were needed in each arm. Blood culture contamination and repeat blood cultures were analyzed with a chi-squared test. Hospital length of stay was analyzed with the t-test. All other secondary outcomes were analyzed with Wilcoxon Rank sum to account for non-normalized distribution.

\section{RESULTS}

The study included a total of 3,331 patients. After review of patient's age, it was confirmed that no patients needed to be excluded due to age. A total of 1,713 patients were included in the pre-ISDD results group and 1,618 patients were included in the ISDD group. Baseline characteristics (except confirming age $\geq 18$ years old) were not obtained as it was predicted to not change the results.

Study Outcome. The primary outcome blood culture contamination rate was $7.47 \%$ in the pre-ISDD group vs. $2.59 \%$ in the ISDD group ( $\mathrm{p}<0.001)$. The per protocol contamination rate was $0.86 \%$. The total hospital cost was $\$ 1,120,000$ in the pre-ISDD group vs. $\$ 383,690$ in the ISDD group, providing a difference of $\$ 736,310$. The per protocol hospital cost analysis was $\$ 138,690$, which provided a difference of $\$ 981,310$. The hospital length of stay $(p=0.7)$, ICU length of stay ( $p=0.3)$, and vancomycin duration of therapy ( $p=0.19)$ were not statistically significant (Figure 1). Vancomycin serum levels obtained were 0.085 vs. 0.075 ( $\mathrm{p}=0.58$ ). Repeat blood cultures were 33 vs. 31 in the two groups, respectively $(\mathrm{p}=0.8)$.

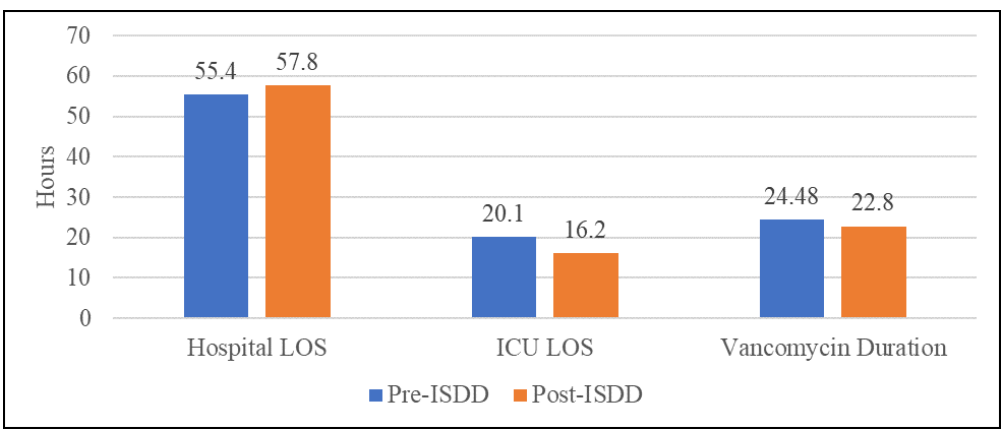

Figure 1. Length of stay and duration of therapy results.

\section{DISCUSSION}

Current recommendations for blood culture contamination are rates less than $3 \%$ set by the CLSI. The baseline AVC ED contamination rate was $6 \%$. Our study demonstrated a statistically significant reduction in blood culture contamination rates by implementing an ISDD. Both post-ISDD groups met the national goal of less than $3 \%$. There was not an $80 \%$ reduction in blood culture contamination in the intent-to-treat analysis (65\% reduction); however, there was over an $80 \%$ reduction in the per protocol analysis ( $88 \%$ reduction). The primary endpoint showed an ISDD, when used appropriately, significantly reduced contamination rates. The difference between the intent-to-treat and per protocol analysis showed the real-life application of using an ISDD 
KANSAS JOURNAL of MEDICINE INITIAL SPECIMEN DIVERSION DEVICE continued.

compared to the true benefit of the device. When the device was being used properly, contamination rates were less than $1 \%$.

Like all novel instruments, compliance can be an issue. Over half of the contaminants in the ISDD group were contributed to nurses not utilizing the ISDD. When it could not be determined if the device was used, it was recorded in the per protocol analysis, meaning the true benefit of the device was likely not captured adequately. Educational instructions for proper ISDD use were provided prior to implementing the device and re-education was done with each nurse when a blood culture contaminant was confirmed throughout the study period. Although re-education was occurring, some nurses simply chose not to use the device. There were no reports of the ISDD being hard to use; however, there were reports that when the ED was busy, nurses went with what they were comfortable with. This reality was known prior to the study which is why a per protocol and intentto-treat analysis were performed to capture the real world effect. However, due to the retrospective nature of this study, the compliance was a major limitation of this study.

The hospital cost analysis showed money potentially saved by using an ISDD. When looking at the per protocol results, almost \$1 million could be saved with consideration to microbiology, antibiotic usage, length of stay, and labor costs. Geisler et al. ${ }^{2}$ showed an average cost accruing to more than $\$ 6,000$ dollars per contaminant with the most influential factors being LOS, daily hospital costs, and antibiotic usage. Many factors influencing the increased costs were avoidable factors. The largest factor was extended length of stay. ${ }^{2}$ This study's estimated cost analysis was unable to find consistency with previous literature regarding cost savings. This study was unable to capture differences within the secondary endpoints, however, there was a significant difference in contamination rates. Based on previously published literature, that difference should have resulted in cost savings. ${ }^{3,4,6,7}$ To date, the use of a studied ISDD is the single most effective intervention for reducing costs related to blood culture contamination. $^{2}$

AVC utilizes rapid diagnostic testing on all blood cultures which allows confirmation of a positive blood culture quicker and time to confirm a contaminant. In the previous studies, rapid diagnostic testing was not used, therefore, they had longer waiting periods for confirmation. This could be a potential answer for the reason as to why this study was unable to detect significant differences in our secondary endpoints and could lead to the conclusion that an ISDD might provide greater benefit in hospitals that do not utilize rapid diagnostic technology for their blood culture analysis.

One of the major limitations of this study was the secondary outcomes analysis took place from the intent-to-treat analysis only. This study could not capture if the ISDD was used with every blood culture obtained. AVC did not require documentation for device use every time a blood culture was drawn. This prevented knowing the true benefit of the device in the secondary outcomes. Future studies should look specifically at secondary outcomes when the ISDD use was confirmed vs. a time period when an ISDD was not used. A recommendation, if implementing an ISDD in a facility, would be to require documentation of whether the device was used.

Another data point not captured within this study was the actual organisms being isolated due to the retrospective nature of this study. This information could provide valuable clinical information when comparing the two study arms, especially if there are profound differences in the organisms being identified. Future studies should include this information, as it potentially could capture trends that clinicians should be aware of going forward.

Nursing turnover between the two study periods could have provided a limitation. Although there was little turnover, there were some nurses included in only one of the time periods. This could change our results as the benefit could have been captured from the nursing staff expertise as opposed to the device itself.

When implementing an ISDD, this study stressed the importance of compliance with the device. Although we were unable to show any statistically significant effect on our secondary outcomes, it was limited by our inability to confirm compliance with the device. As shown through the primary outcome, contamination rates were improved when the ISDD usage was confirmed. ISDD implementation will have the biggest benefit when there is near $100 \%$ compliance.

\section{CONCLUSIONS}

This study showed that utilizing an ISDD significantly reduced blood culture contamination. The study also showed that when implementing the ISDD to a healthcare system, compliance is important and will affect contamination rates dramatically. AVC ministries final decision was to continue using the ISDD due to the proven benefit in reducing blood culture contamination. Although it was shown that barriers of compliance can reduce the benefit of an ISDD, with continuing re-education and increased compliance, contamination rates were expected to decline.

\section{ACKNOWLEDGEMENTS}

The authors thank Kenneth Utz, Pharm.D., BCOP and Connie Schaefer, RN, CIC, for their assistance with data collection, and Scott Taylor, Pharm.D., MSc, BCPS for his assistance with data analysis.

\section{REFERENCES}

${ }^{1}$ Bell M, Bogar C, Plante J, Rasmussen K, Winters S. Effectiveness of a novel specimen collection system in reducing blood culture contamination rates. $\mathrm{J}$ Emerg Nurs 2018; 44(6):570-575. PMID: 29685676.

${ }^{2}$ Geisler BP, Jilg N, Patton RG, Pietzsch JB. Model to evaluate the impact of hospital-based interventions targeting false-positive blood cultures on economic and clinical outcomes. J Hosp Infect 2019; 102(4):438-444. PMID: 30928573.

${ }^{3}$ Bekeris LG, Tworek JA, Walsh MK, Valenstein PN. Trends in blood culture contamination: A College of American Pathologists Q-Tracks study of 356 institutions. Arch Pathol Lab Med 2005; 129(10):1222-1225. PMID: 16196507.

${ }^{4}$ Skoglund E, Dempsey CJ, Chen H, Garey KW. Estimated clinical and economic impact through use of a novel blood collection device to reduce blood culture contamination in the emergency department: A cost-benefit analysis. J Clin Microbiol 2019; 57(1):e01015-01018. PMID: 30355758.

${ }^{5}$ Hall K, Lyman JA. Updated review of blood culture contamination. Clin Microbiol Rev 2006; 19(4):799-802. PMID: 17041144.

${ }^{6}$ Gander RM, Cavuoti D, Alatoom A, et al. Impact of blood cultures drawn by phlebotomy on contamination rates and health care costs in a hospital emergency department. J Clin Microbiol 2009; 47(4):1021-1024. PMID: 19171686. 
${ }^{7}$ Garcia RA, Spitzer ED, Beaudry J, et al. Multidisciplinary team review of best practices for collection and handling of blood cultures to determine effective interventions for increasing the yield of true-positive bacteremia, reducing contamination, and eliminating false-positive central line-associated bloodstream infections. Am J Infect Control 2015; 43(11):1222-1237. PMID: 26298636.

${ }^{8}$ Arenas M, Lukey J, Boseman G, Navarathna D. The impact of blood culture diversion devices on contamination rates. January 2, 2019. https://www. kurin.com/the-impact-of-blood-culture-diversion-devices-on-contamination-rates/.Accessed July 22, 2020.

${ }^{9}$ Rupp ME, Cavalieri RJ, Marolf C, Lyden E. Reduction in blood culture contamination through use of initial specimen diversion device. Clin Infect Dis 2017; 65(2):201-205. PMID: 28379370.

Keywords: blood culture, emergency department, infection control, infections
KANSAS JOURNAL of MEDICINE

INITIAL SPECIMEN DIVERSION DEVICE

continued. 